\title{
Eficacia de un programa psicoedu- cativo para cuidadores informales de adultos mayores con demencia
}

\section{Efficacy of a pyschoeducational program for informal caregivers of older adults with dementia}

Ana Margarita Espín Andrade ${ }^{1}$
Palabras clave: psicoeducativo, cuidadores, demencia, adultos mayores

Key words: pyshoeducationd, caregivers, dementia, older adults

\section{Resumen}

Objetivos: diseñar y evaluar la eficacia de un programa psicoeducativo para cuidadores informales de adultos mayores con demencia.

Métodos: se realizó un diseño de desarrollo tecnológico. Se seleccionó una muestra aleatoria estratificada de 96 cuidadores de servicios gerontológicos de ciudad de La Habana, que constituyeron los casos, los restantes 96 cuidadores integraron el grupo control. Para el diseño del programa se identificaron las necesidades de aprendizaje de los cuidadores. El programa constó de 12 sesiones, llevadas a cabo por un equipo multidisciplinario. Se aplicaron técnicas afectivo-participativas y estudio de casos.

Para la evaluación de la eficacia del programa se siguió el modelo de evaluación de programas de Donabedian.

Resultados: la diferencia entre el grupo de estudio y control antes de la intervención no era significativa con relación a los conocimientos acerca de la enfermedad, las habilidades para el cuidado de los ancianos, la calidad del cuidado ofrecido, el consumo de medicamentos por el cuidador, la presencia de trastornos conductuales en el enfermo y la carga del cuidador, mientras que después de la intervención, se observó un cambio favorable altamente significativo de todas estas variables.

Hubo un aumento de los casos sin depresión después de la intervención aunque no se evidenció una diferencia significativa entre el grupo estudio y control.

El 82,3\% de los cuidadores reportaron una elevada satisfacción con el programa.

Conclusiones: el programa fue eficaz debido a que aumentaron los conocimientos y habilidades para el cuidado, disminuyó la carga así como la cantidad de medicamentos consumidos por el cuidador, mejoró la conducta del enfermo y la calidad del cuidado ofrecido.

\section{Abstract}

Objectives: to design and assess the efficacy of pyschoeducational program for informal caregivers of older adults with dementia.

Methods: it was made a technological development design. A stratified random sample of 96 caregivers of gerontological services from Havana City who made up the target cases. The remaining 96 caregivers were part of the control group. For the program design, the caregivers' learning needs were determined. The 12-session program was carried out by an interdisciplinary team. Affective-participative techniques and case study were applied.

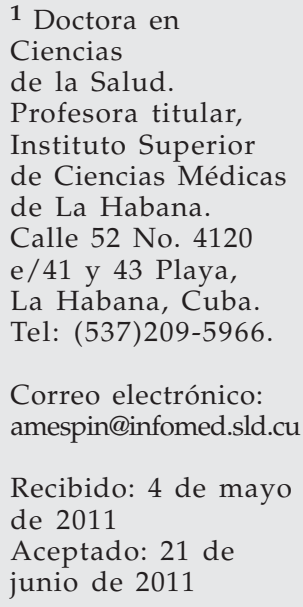


For the evaluation of program efficacy it was followed the Donabedian's program assessment model

Results: before the intervention the difference between the study group and the control group was not significant regarding knowledge about the illness, the skills to take care of elderly, the quality of care provided, drug taking by caregiver, the presence of behavioral disorders in the patient and the caregiver's burden. On the contrary, after the intervention it was observed a highly significant favorable change in all these variables.

There was an increase in free-depression cases after the intervention; although there was not significant difference between the study and control group.

$82,3 \%$ of caregivers were very satisfied with the program.

Conclusions: the program proved to be effective since it increased knowledge and skills for care, decreased the work burden, as well as the amount of drugs taken by the caregiver. It also improved the patient behavior and the quality of the care provided.

\section{Introducción}

El envejecimiento de la población es sin duda, la principal característica demográfica de Cuba en la actualidad, y también perspectivamente. Ese es el efecto principal de la transición de la fecundidad y obviamente, la sociedad tendrá que adecuar sus acciones, desde el punto de vista de su funcionamiento económico, social y cultural ${ }^{(1)}$.

En el año 2008, el 17\% de la población cubana correspondía a personas mayores de sesenta años. Este comportamiento debe intensificarse; para el año 2050 se pronostica que el 37,3\% de la población será adultos mayores, Cuba tendrá todas las características de un país envejecido, y se situará entre los 11 países del mundo de mayor población de ancianos ${ }^{(2)}$.

El crecimiento del grupo de edad de ochenta y más años, junto al cambio en los patrones epidemiológicos de los ancianos que se centra en problemas crónicos y degenerativos, está propiciando que cada vez sea mayor en el mundo el número de personas que presentan algún tipo de dependencia (3).

Una de las afecciones que más limita las actividades de la vida diaria del adulto mayor, es la demencia. Esta enfermedad provoca alteraciones no sólo en el enfermo sino también en el cuidador y en la familia en general, ya que su aparición demanda de una redistribución de los roles familiares, y genera un elevado estrés por las múltiples manifestaciones conductuales y el desconocimiento del proceso que las ocasiona, entre otros factores ${ }^{(4)}$.

La ausencia de una terapéutica para $\mathrm{cu}^{-}$ rar la demencia y para detener la pérdida de las capacidades y funciones del enfermo, hace imprescindible el desarrollo de tratamientos alternativos, como es el caso de la intervención en la familia de una manera precoz con vistas a mejorar los cuidados, lo cual contribuye a lograr una mejor evolución del enfermo.

Es insuficiente la información y educación que se brinda a la población y en particular, a la familia acerca de las demencias y el cuidado y manejo del paciente que la sufre, por ser bastante reciente el aumento de su prevalencia y el consecuente impacto social y económico.

Las intervenciones tienen como objetivo disminuir la carga que experimentan las personas que cuidan ancianos dependientes, el estrés y malestar asociados al cuidado, y por otra parte, mejorar la calidad de los cuidados que reciben estos ancianos. Para conseguir tales objetivos, se han empleado procedimientos de intervención psicosocial tales como grupos de educación e información, grupos de apoyo emocional y autoayuda, grupos de entrenamiento en técnicas cognitivo conductuales, así como también tratamientos individuales como asesoramiento familiar, consulta familiar o terapia psicológica individual ${ }^{(5)}$. 
Los programas psicoeducativos tienen el propósito de ofrecer, en primer lugar, información acerca de la enfermedad y su manejo, así como brindar estrategias de afrontamiento y apoyo emocional a los cuidadores. Se ha evidenciado que los grupos psicoeducativos pueden evitar comportamientos, cogniciones y emociones desadaptativas en la relación cuidador-enfermo ${ }^{(6)}$.

Los programas psicoeducativos son el procedimiento de intervención más habitual y generalizado para dotar a los familiares de recursos para el cuidado. Algunos de los beneficios que se han encontrado con la aplicación de estos programas son que mejoran la calidad del cuidado, disminuyen los problemas conductuales y el validismo del enfermo, disminuyen el consumo de medicamentos de este, mejoran la adaptación del cuidador a la nueva situación y disminuyen los problemas emocionales del cuidador y del paciente, entre otros.

Se coincide con la afirmación acerca de que los programas que tan solo brindan información a los cuidadores, pueden generarles un alto grado de ansiedad, lo cual se ha relacionado con una anticipación de la pérdida y con el conocimiento del alcance de la enfermedad, por lo que es necesario ofrecerles ayuda para procesar la información, de tal forma que sus emociones sean más adecuadas ${ }^{(7)}$.

La mayoría de los estudios de intervención psicoeducativa se han realizado en grupos de cuidadores de personas con demencia, aunque se reportan algunos en otras enfermedades crónicas como la esquizofrenia, la insuficiencia renal crónica y otras afecciones en las que el conocimiento y el manejo adecuado del cuidador familiar es imprescindible por el estrés que les provocan ${ }^{(8,9)}$.

Son múltiples las propuestas para intervenir con estos cuidadores familiares, pero muy pocas las que se han realizado en Latinoamérica y el Caribe, y menos aún aquellas que han sido evaluadas con rigor ${ }^{(10)}$.

Un meta-análisis que encontró 54 estudios de intervención psicoeducativa en cuidadores de personas con demencia, señaló que 40 de ellos se realizaron en los Estados Unidos ${ }^{(11)}$. No obstante, se advierten algunos programas de intervención en esta región, como por ejemplo "Cuidando al cuidador", en Colombia(12) y la llamada "Escuela de cuidadores" en Cuba(13), estos constituyen serios intentos de mejorar la calidad de vida del binomio cuidador-paciente.

En Cuba se realizan diferentes intervenciones psicoeducativas en grupos de cuidadores en algunas localidades, sin embargo, no se recogen estudios que evalúen su eficacia, lo cual es imprescindible porque permite brindar una ayuda y preparación de mayor calidad al cuidador.

La eficacia se refiere a los resultados en relación con las metas y cumplimiento de los objetivos. Para ser eficaz se deben priorizar las tareas y realizar ordenadamente aquellas que permiten alcanzarlos mejor; es el grado en que un procedimiento o servicio puede lograr el mejor resultado posible; es la relación objetivos/resultados bajo condiciones ideales ${ }^{(14)}$.

Para lograr la mayor eficacia de este tipo de intervenciones se deben tener en cuenta una serie de elementos:

- Adecuarlos a las necesidades de los cuidadores.

- Realizar un trabajo multidisciplinario.

- Realizar la intervención precozmente.

- Equilibrar los componentes informativos, emocionales y habilidades del cuidador.

- Integrarlos a los servicios sociales y sanitarios de la comunidad.

Existen pocas propuestas de intervenciones psicoeducativas para los cuidadores de ancianos en los países en desarrollo, y algunas de ellas no cuentan con suficiente rigor metodológico, por lo que es necesario basarse en modelos teóricos para implementarlas y probar su eficacia. La autora selecciona el modelo teórico de afrontamiento y estrés adaptado al cuidado, y propone su modificación al concebir una relación de retroalimentación entre los elementos que lo conforman.

\section{Material y Método}

Se realizó un diseño de desarrollo tecnológico, pues se creó una estrategia para la 
intervención en cuidadores informales de adultos mayores con demencia.

Del total de 192 cuidadores correspondientes al universo, se seleccionó una muestra aleatoria estratificada de 96 cuidadores que constituyeron los casos, los restantes 96 cuidadores integraron el grupo control.

Se observó que en ambos grupos la edad de los pacientes que prevaleció fue de 75 a 84 años (43,7\%); en ambos la mayoría de los pacientes eran del sexo femenino para un $81,2 \%$, más de la mitad presentaban enfermedad de Alzheimer (58,3\% y 56,3\%) y primó en los dos grupos la etapa moderada de la enfermedad $(54,2 \%$ y $53,1 \%)$. Con respecto a los datos de los cuidadores de cada uno de los grupos (estudio y control) se aprecia que la edad que prevaleció fue de 40 a 59 años (56,3\% y 55,2\%); el sexo femenino en ambos fue del $81,2 \%$; el estado conyugal casado $(63,5 \%$ y $54,1 \%)$, el nivel educacional universitario $(33,3 \%$ y $35,4 \%)$; con relación al vínculo laboral en ambos grupos la mayoría no trabajaba $(42,7 \%$ y $46,9 \%)$ y el parentesco con el enfermo fueron los hijos en los dos grupos (71,9\% y 67,8\%).

Se observó un porcentaje similar de cuidadores con presencia de carga en ambos grupos ( $50 \%$ y $47,1 \%)$.

Se recogió el consentimiento informado de cada uno de los cuidadores del grupo de estudio, donde se explicaron los objetivos de la investigación, el carácter voluntario de su participación y demás aspectos contemplados en la Declaración de Helsinki(15).

Por la implicación ética que conllevaba esta selección, se le explicó nuevamente al grupo control las razones de su exclusión y se recordó el compromiso de no participar en ningún tipo de intervención dentro de los tres meses que duraba el programa, que fue recogido en el Acta 1 de consentimiento informado.

Para el diseño del programa se identificaron las necesidades de aprendizaje (sentidas y normativas) a través de un método de búsqueda de consenso, específicamente el método de informadores clave. Para las normativas se aplicó además el "Test de conocimientos y habilidades del cuidador" a los 75 cuidadores seleccionados para la identificación de necesidades sentidas. Esta es una prueba diseñada por la autora del trabajo y validada en el CITED para medir los conocimientos y las habilidades con que cuentan los cuidadores familiares de los enfermos que sufren demencia. Consta de 14 preguntas, las que miden las siguientes variables: nombre de la enfermedad, característica principal, pronóstico, tratamiento, aseo personal, comunicación con el enfermo, accidentes en el hogar, alimentación, extravío del enfermo, incontinencia, manejo de la agresividad, hábitos de sueño, actividades físicas y sociales y sobrecarga del cuidador ${ }^{(16)}$.

Se consideró pertinente, además de brindar conocimientos acerca de la enfermedad y el desarrollo de habilidades para el cuidado, incluir aspectos relacionados con el rol del cuidador, al velar por el desarrollo de estrategias de afrontamiento adecuadas, el logro de un mayor control emocional de los cuidadores y hacerlo consciente de la necesidad de su propio cuidado para garantizar un cuidado de mayor calidad.

Se confeccionó un programa de 12 sesiones con una frecuencia semanal y una duración de una hora y media cada sesión, donde cada día se trató un tema diferente, dirigido por un especialista del equipo multidisciplinario (médico, psicólogo, enfermera, trabajadora social, defectólogo, fisioterapeuta). La sesiones se realizaron a través de métodos participativos e interactivos con técnicas afectivo-participativas y estudio de casos.

Se consideró importante realizar la intervención en tres áreas de salud de los cuidadores, ya que estas intervenciones deben ser implementadas donde sean más accesibles para el cuidador, pues éste dispone de poco tiempo, y es este uno de los grandes problemas de los que han adolecido los programas psicoeducativos para cuidadores.

Se realizó la capacitación de todos los equipos multidisciplinarios de cada una de las áreas seleccionadas para la intervención, de manera que el programa se aplicara de la manera más homogénea posible, al utilizarse los mismos medios y técnicas (demostrativa, expositiva, con un predominio de las participativas). La capacitación comprendió tanto la parte del programa como la evaluación. 
Para la evaluación de la eficacia del programa se siguió el modelo de evaluación de Donabedian: estructura, proceso y resultados $^{(17)}$. Para la formulación de los criterios explícitos de evaluación se aplicó la metodología del propio autor, aplicándose la técnica de grupo nominal con diez expertos. Se utilizaron pruebas psicológicas, cuestionarios y guías de observación, para medir los criterios seleccionados, donde algunos de ellos estaban validados en el país y otros fueron validados para el presente estudio.

Los criterios y escalas utilizados en la evaluación de resultados fueron:
- Conocimientos acerca de la enfermedad y su manejo: test de conocimientos y habilidades del cuidador ${ }^{(16)}$.

- Habilidades para el cuidado: test de conocimientos y habilidades del cuidador ${ }^{(16)}$.

- Sentimiento de carga del cuidador: test de carga de Zarit ${ }^{(18)}$.

- Grado de depresión del cuidador: test de depresión de Beck ${ }^{(19)}$.

- Consumo de fármacos (cuidador): entrevista estructurada.

- Percepción de trastorno de conducta del paciente: inventario neuropsiquiátrico ${ }^{(20)}$.

- Grado de satisfacción con el programa: cuestionario de satisfacción.

- Calidad del cuidado al enfermo: guía de observación.

\begin{tabular}{|c|c|c|}
\hline Criterios & Escala de clasificación & Descripción de la escala \\
\hline \multirow{2}{*}{$\begin{array}{l}\text { Conocimientos acerca } \\
\text { de la enfermedad y su } \\
\text { manejo }\end{array}$} & Suficientes & $\begin{array}{l}70 \% \text { o más de las preguntas } 1,2,3,4 \text { del test de conocimientos } \\
\text { y Habilidades del Cuidador correctas. }\end{array}$ \\
\hline & Insuficientes & Menos del 70\% de las preguntas 1, 2, 3, 4 del test correctas \\
\hline \multirow[t]{2}{*}{$\begin{array}{l}\text { Habilidades para el } \\
\text { cuidado }\end{array}$} & Suficientes & $\begin{array}{l}70 \% \text { o más de las preguntas de la } 5 \text { a la } 14 \text { del test de } \\
\text { conocimientos y habilidades del cuidador correctas }\end{array}$ \\
\hline & Insuficientes & $\begin{array}{l}\text { Menos del } 70 \% \text { de las preguntas de la } 5 \text { a la } 14 \text { del test } \\
\text { correctas }\end{array}$ \\
\hline \multirow{4}{*}{$\begin{array}{l}\text { Sentimiento de carga } \\
\text { del cuidador }\end{array}$} & Ausente & Menos de 47 puntos en el Test de carga de Zarit \\
\hline & Leve & de 47 a 64 \\
\hline & Moderada & de 65 a 80 \\
\hline & Severa & de 81 a 84 \\
\hline \multirow{4}{*}{$\begin{array}{l}\text { Grado de depresión } \\
\text { del cuidador }\end{array}$} & Ausente & Menos de 7 en el Test de Depresión de Beck \\
\hline & Leve & de 7 a 18 \\
\hline & Moderada & de 18 a 24 \\
\hline & Severa & de 2432 \\
\hline \multirow[t]{2}{*}{$\begin{array}{l}\text { Calidad del cuidado } \\
\text { al paciente }\end{array}$} & Adecuada & $\begin{array}{l}\text { Al menos } 6 \text { aspectos de la guía de observación del cuidado en } \\
\text { el hogar evaluados de buenos. }\end{array}$ \\
\hline & Inadecuada & $\begin{array}{l}\text { Menos de } 6 \text { aspectos de la guía de observación del cuidado en } \\
\text { el hogar evaluados de buenos. }\end{array}$ \\
\hline \multirow{4}{*}{$\begin{array}{l}\text { Consumo de } \\
\text { fármacos del } \\
\text { cuidador }\end{array}$} & Ausente & No consume \\
\hline & Ligero & Consume 1 o 2 fármacos al día \\
\hline & Moderado & Consume 3 o 4 fármacos al día \\
\hline & Severo & Consume más de 4 al día \\
\hline \multirow{5}{*}{$\begin{array}{l}\text { Percepción de } \\
\text { trastorno de conducta } \\
\text { del paciente }\end{array}$} & & Según el Inventario Neuropsiquiátrico \\
\hline & Ausente & Si tiene 0 en todos los ítems \\
\hline & Leve & Si tiene 1 o 2 en al menos un ítem \\
\hline & Moderado & Si tiene entre 3 y 6 en al menos un ítem \\
\hline & Severo & Si tiene entre 8 y 12 en al menos un ítem \\
\hline \multirow[t]{3}{*}{$\begin{array}{l}\text { Grado de satisfacción } \\
\text { con el programa }\end{array}$} & Elevado & $\begin{array}{l}\mathrm{Si} \text { responde afirmativamente a las } 3 \text { preguntas del } \\
\text { cuestionario }\end{array}$ \\
\hline & Moderado & Si responde afirmativamente a 2 preguntas del cuestionario \\
\hline & Bajo & Si responde afirmativamente a 1 pregunta del cuestionario \\
\hline \multirow[t]{2}{*}{$\begin{array}{l}\text { Momento en relación } \\
\text { con la intervención }\end{array}$} & Antes & $\begin{array}{l}\begin{array}{l}\text { Resultados en los indicadores anteriores previo a la } \\
\text { intervención }\end{array} \\
\end{array}$ \\
\hline & Después & $\begin{array}{l}\text { Resultados en los indicadores anteriores al culminar la } \\
\text { intervención. }\end{array}$ \\
\hline
\end{tabular}


Para identificar las posibles diferencias existentes entre el grupo de estudio y el grupo control, antes y después de la intervención, con relación a las variables cualitativas analizadas, se emplearon tablas de contingencia $(2 \times 2)$ por tratarse de variables dicotómicas en su mayoría, y se aplicó la prueba de Homogeneidad $\mathrm{X}^{2}$, con un nivel de confiabilidad del $95 \%$.

\section{Resultados}

\section{Evaluación de estructura}

Con relación a los recursos humanos, hubo un $100 \%$ de disponibilidad, pues se contó para la intervención con equipos completos de profesionales calificados y con amplia experiencia, integrados por geriatras, médicos generales integrales, enfermeras, psicólogos, trabajadores sociales, defectólogos y técnicos en fisioterapia. El personal de las áreas de salud eran integrantes de los EMAG, y todos habían cursado maestrías o diplomados en Geriatría y Gerontología. Por todo esto, la competencia fue valorada como adecuada, ya que el $100 \%$ de los especialistas contaban con la calificación requerida. Las sesiones se realizaron en dos locales, uno para los grupos del CITED y el Policlínico Moncada, y el otro en el Museo de Playa, para los grupos de las áreas de salud de dicho Municipio. Ambos disponían del 100\% de condiciones materiales óptimas, de ventilación, iluminación y confort. Se contó con los medios de enseñanza planificados, en el 100\% de las sesiones, al contar con datashow, computadora, pizarra y pancartas, los que tenían una calidad adecuada, que aseguró el desarrollo satisfactorio de las intervenciones.

Con respecto a la evaluación de los expertos acerca del diseño del programa de intervención, se observó que el 100\% encontró los contenidos formulados claramente, que los temas incluidos eran los correctos, apreciaron una elevada pertinencia del programa, el 90\% consideró que existía congruencia entre los objetivos y los temas, y planteó como adecuadas las técnicas seleccionadas para cada actividad; el $80 \%$ enten- dió que doce sesiones era la cantidad adecuada dados los objetivos del programa. Por todo esto se valoró como adecuada la calidad del diseño.

\section{Evaluación de proceso}

Se cumplió con la asistencia del $80 \%$ de los cuidadores a las sesiones, que se correspondió con el estándar fijado. Esto se consideró un éxito de la intervención, pues resultaba difícil para ellos asistir sistemáticamente a estos grupos, por el poco tiempo disponible, y por el hecho de tener que dejar a los pacientes bajo el cuidado de otras personas. Por esta razón, en muchos casos los cuidadores llevaban a los enfermos a los encuentros "por no tener con quién dejarlos", y un miembro del equipo asumía la atención de los mismos en otro local, mientras se desarrollaba la sesión.

El 100\% de las sesiones tuvo una calidad satisfactoria, al tener en cuenta la participación de los cuidadores y el dominio de las técnicas por el especialista.

El manejo del tema por el profesional fue evaluado como adecuado en el $100 \%$ de las sesiones: se observó un amplio dominio del contenido, que se manifestaba en las respuestas acertadas de los especialistas a las diferentes inquietudes de los integrantes.

En el 100\% de las sesiones se estableció una dinámica adecuada, existió una buena interacción entre los miembros de los grupos donde los cuidadores se brindaban consejos y soluciones a los diferentes problemas basados en su propia experiencia, existía buena comunicación entre ellos, espíritu de apoyo y de solidaridad en todo momento.

\section{Evaluación de resultados}

La diferencia entre el grupo de estudio y el de control con relación a los conocimientos acerca de la enfermedad antes de la intervención no era significativa $(p=0,8)$; se apreció una diferencia significativa entre ambos grupos posterior a la intervención ( $p$ $=0,0$ ), donde aumentó el conocimiento suficiente sobre la enfermedad de un $18,7 \%$ a un $90,6 \%$ en el grupo de estudio, mientras no se observaron diferencias en el grupo control. 
Con relación a las habilidades de cuidado, no se observó diferencia significativa entre el grupo de estudio y control antes de la intervención $(p=0,11)$; mientras que después de la intervención, se observó un aumento significativo (de un $5 \%$ a un $90 \%$ ) de las habilidades para el cuidado de los ancianos en el grupo de estudio con respecto al grupo control, que se evidencia en la $\mathrm{p}=0,0$.

Con relación a la carga, no existían diferencias significativas entre el grupo de estudio y control antes de la intervención, lo que se evidencia en la p de 0,6 . Sin embargo, se aprecia que después de la intervención, hubo una variación significativa $(\mathrm{p}=$ 0,01 ) al disminuir la carga del grupo de estudio con respecto al control, de un $50 \%$ que no tenía carga a un 70, 9\%. También se modificó favorablemente el grado de la carga, se observan diferencias en el grupo de estudio, donde la carga leve disminuyó de un $38,5 \%$ a un $20,8 \%$, mientras que en el grupo control no existió variación.

Hubo un aumento de los casos sin depresión después de la intervención en el grupo de estudio con respecto al control, de un $26 \%$ a un $57,3 \%$, aunque no se evidenció una diferencia significativa entre el grupo de estudio y control, ni antes ni después de la intervención, lo que se expresó en los valores de $\mathrm{p}$ mayores de 0,05 . Se apreció una variación favorable del grado de depresión en el grupo de estudio, al disminuir la depresión leve de $51 \%$ al $30 \%$, pero la diferencia con el grupo control no fue significativa, lo que indicó que no se redujo lo suficiente con la intervención.

Con respecto a la calidad del cuidado, no existían diferencias significativas entre el grupo de estudio y control antes de la intervención; sin embargo, después de esta, la diferencia se hizo notable entre ambos grupos al aumentar la calidad adecuada en el grupo de estudio, de $8,3 \%$ a $95,8 \%$, no así en el grupo control, lo que se corrobora con el valor de $\mathrm{p}$ de 0,0 .

Existía un elevado uso de medicamentos por parte de ambos grupos de cuidadores, y no se evidenció diferencia significativa entre el grupo de estudio y control antes de la intervención $(p=1)$. Sin embargo, después de la intervención hubo una diferencia significativa entre ambos grupos en el consumo de fármacos $(p=0.0)$ al aumentar el número de cuidadores que no consumía medicamentos de 23 a 61 en el grupo de estudio. También se redujo el número de medicamentos a consumir, es decir, el grado de consumo de fármacos: el grado leve de 44 a 24 , el grado moderado de 17 a 11 y el severo de 12 a 0 .

No existían diferencias significativas en el grupo de estudio con respecto al control en cuanto a la percepción de los trastornos de conducta $(p=0,8)$ antes de la intervención; sin embargo, estas diferencias fueron notorias después de la intervención $(p=0,0)$.

Antes de la intervención todos los pacientes presentaban trastornos conductuales según la percepción del cuidador, sin embargo, después existían nueve pacientes que no presentaron trastornos conductuales; así también mejoró el grado de severidad de dichos trastornos al descender de 62 a 26 pacientes con trastorno de conducta moderado a leve en el grupo de estudio, mejoría que no se evidenció en el grupo control $(p=0,0)$.

El 82,3\% de los cuidadores reportaron una elevada satisfacción con el programa, un $16,7 \%$ una satisfacción moderada y sólo el $1 \%$ presentó un bajo grado de satisfacción.

\section{Discusión}

Si bien transmitir conocimientos no debe ser el único objetivo de estos programas para cuidadores, es fundamental para ellos conocer acerca de las características de la enfermedad, de su tratamiento, del pronóstico, así como de los problemas de conducta y síntomas psíquicos, y cómo actuar ante cada uno de ellos; esto permite un manejo más adecuado, aumenta la seguridad del cuidador y disminuye su nivel de ansiedad.

Este tipo de intervención se ha demostrado que aumenta los conocimientos del cuidador, así por ejemplo, en el estudio de Martín y col., se obtuvo una mejora en el conocimiento del manejo de los cambios posturales del enfermo ${ }^{(21)}$.

Estudios realizados en los Estados Unidos en el año 2004, han constatado el incre- 
mento del nivel de conocimientos acerca de la enfermedad y su cuidado(22, 23).

Estos resultados coinciden con una reciente revisión sobre intervenciones psicoeducativas, que permitió conocer que los cuidadores informales de personas con demencia pueden aprender habilidades y estrategias dirigidas a reducir la aparición, frecuencia o la intensidad de comportamientos problemáticos asociados a la demencia(24).

Crespo y col. han planteado que la aparición de problemas emocionales en el cuidador depende más de las propias habilidades y recursos del cuidador, que de la problemática específica que presenta la persona cuidada $^{(25)}$. Por esta razón es muy importante además de trasmitir información, desarrollar habilidades adecuadas para el cuidado.

Schumcher y col. plantearon en el año 2000 que "el cuidado en casa requiere que los cuidadores tengan destrezas y conocimientos cada vez más complejos"(26).

Mittelman y col. en un trabajo realizado en el año 2004 planteó que "las intervenciones psicosociales no pueden aliviar aspectos dolorosos inherentes a la situación, tales como los sentimientos de pérdida experimentados por los cuidadores. Existen diferentes razones por las que la depresión u otras variables relativas al malestar emocional pueden no ser indicadores sensibles al cambio en las intervenciones, entre ellas está la amenaza que acompaña al conocimiento de la naturaleza deteriorante del curso de la demencia(27).

A tono con estos resultados, un programa psicoeducativo encaminado a disminuir los sentimientos negativos de los cuidadores, mostró que los sentimientos que mejoraron fueron la ira, la angustia y el miedo, mientras que la desesperanza, que es el sentimiento que se encuentra en la base de la depresión, apenas sufrió cambio ${ }^{(28)}$.

López y Crespo en el 2007 señalaron que son mejores los resultados en la disminución de la ira, la hostilidad y la ansiedad, que en la modificación de los niveles de depresión, al ser esta última la única medida que en ciertos estudios se incrementa. Dado que cuidar es un estresor crónico, cuya pre- sencia se mantiene durante y después de la intervención, no es de extrañar que sea difícil modificar el malestar ${ }^{(29)}$.

Sin embargo, otros programas han demostrado su eficacia para disminuir de forma significativa la depresión y otras alteraciones emocionales del cuidador, como la ansiedad y la ira ${ }^{(30-32)}$. Al parecer, incide en esta mejoría del estado emocional del cuidador, la reducción de pensamientos disfuncionales que se producen por la intervención ${ }^{(33)}$.

El aumento en la calidad del cuidado al enfermo se explica por la mejoría de los diferentes aspectos del cuidado que fueron observados, como son: el aspecto higiénicopersonal del paciente, la comunicación con el paciente, las condiciones para la movilidad del enfermo en el hogar, la estimulación física, psicológica y social al enfermo, y el cumplimiento de medidas de seguridad para evitar accidentes.

Los resultados de otro programa de intervención reportaron un cambio de actitud de los cuidadores y la mejora en la calidad de la relación entre los cuidadores y los enfermos ${ }^{(34)}$.

El menor consumo de medicamentos posterior a la intervención se pudiera explicar porque al mejorar su bienestar, el cuidador tiene que recurrir menos al medicamento, pues mejoran aspectos subjetivos relacionados con el cuidado y se logra un mayor cuidado de sí mismo y de su salud.

Si bien el presente estudio no incluyó la evaluación de otras variables de la salud del cuidador después de la intervención, se ha comprobado que este tipo de programas mejora la salud general y percibida de los cuidadores $^{(35,36)}$.

La mejoría de los problemas de conducta de los enfermos pudiera explicarse porque los cuidadores comprenden que los problemas conductuales son parte del cuadro clínico de la enfermedad y por tanto, su valoración de estos comportamientos, así como sus expectativas con relación a eliminarlos, se modifica adecuadamente. Además, incide que aprenden a manejar correctamente los diferentes trastornos de conducta, lo que les posibilita encontrar soluciones a corto plazo, lo que va mejorando 
el comportamiento general del enfermo. Por otra parte, la mejoría del estado emocional del cuidador hace más "tolerable" la conducta de la persona cuidada.

Otros programas de intervención han demostrado su efectividad para disminuir los problemas conductuales de las personas cuidadas, y han observado diferencias significativas en la disminución de la irritabilidad, los delirios y las alucinaciones ${ }^{(37)}$.

La participación de los cuidadores en programas psicoeducativos puede tener efectos positivos para las personas cuidadas, ya que puede disminuir la frecuencia y/o intensidad de problemas de conducta asociados a la demencia(38).

En un estudio realizado en 158 cuidadores de pacientes con demencia que participaron en un grupo psicoeducativo, el $14 \%$ disminuyó sus reacciones emocionales negativas a los trastornos conductuales del paciente, en comparación con un 5\% del grupo control. Además, fue significativa la diferencia con respecto a la presencia de problemas conductuales en el paciente ${ }^{(39)}$.

El hecho de que la mayoría de los cuidadores estuvieron satisfechos con la intervención demuestra que se cumplieron sus expectativas, quizás incluso por encima de lo esperado en la gran mayoría de ellos, lo que es explicable, pues todos los parámetros evaluados mejoraron después de la intervención. Estos resultados coinciden con los hallados por García y col. en el 2005 en los cuidadores que participaron en un programa de intervención, donde el $75 \%$ de ellos se sintieron bastante satisfechos ${ }^{(40)}$.

A manera de conclusiones se puede decir que la evaluación satisfactoria tanto de la estructura como del proceso de la intervención, garantizó en alguna medida el éxito en la evaluación de los resultados, pues el hecho de asegurar la calidad óptima de la intervención desde el comienzo y durante el desarrollo de las sesiones, permitió el logro de los objetivos propuestos y demostrar la eficacia del programa de intervención.

Se tomaron en cuenta además una serie de aspectos que hicieron posible la eficacia del programa, estos son: las intervenciones se

$$
\text { ajustaron a un marco teórico y }
$$

metodológico riguroso, y se partió de la evaluación de las necesidades de aprendizaje de los cuidadores. También se dedicó una buena parte del programa al entrenamiento de habilidades, tanto para satisfacer las necesidades básicas del enfermo como para afrontar los comportamientos inadecuados del paciente y manejar el propio estrés.

El programa fue eficaz debido a que aumentaron los conocimientos y habilidades para el cuidado, disminuyó la carga, así como la cantidad de medicamentos consumidos por el cuidador, mejoró la conducta del enfermo y la calidad del cuidado ofrecido, observándose un alto grado de satisfacción de los cuidadores con la intervención realizada.

\section{Referencias Bibliográficas}

1. Alfonso JC. 2006. El descenso de la fecundidad en Cuba: de la primera a la segunda transición demográfica. Rev Cubana Salud Pública 32(1):1-19.

2. Alfonso JC. 2009. El Impacto Social del Envejecimiento Demográfico: el caso Cuba. Simposio Internacional "Carlos Font Pupo". La Habana, Septiembre de 2008. http:// www.one.cu

3. Pérez VT. 2005. El deterioro cognitivo: una mirada previsora. Rev Cubana Med Gen Integr (21):1-2.

4. Dueñas E, Martínez MA, Morales B, Muñoz C, Viáfara, AS, Herrera JA. 2006. Síndrome del cuidador de adultos mayores discapacitados y sus implicaciones psicosociales. Colomb Med 37(2) supl.1:1-7.

5. Muela JA, Torres CJ, Peláez EM. 2002. Comparación entre distintas clasificaciones de las estrategias de afrontamiento en cuidadores de enfermos de Alzheimer. Psicothema 14(3):558-563.

6. Zambrano R, Ceballos P. 2007. Síndrome de carga del cuidador. Rev Colomb Psiquiatr 36(suppl.1):26-39.

7. Sorensen S, Pinquart M, Duberstein P. 2002. How effective are interventions with caregivers? An update meta-análisis. The Gerontologist 42(3):356-37.

8. Cassidy E, Hill S, Callaghan O. 2002. Eficacia de una intervención psicoeducativa en la mejora del conocimiento de los familiares sobre la esquizofrenia y la reducción de nuevas hospitalizaciones. Eur Psychiatry Ed. Esp. 9:117-122. 
9. Bañobre A, Vázquez J, Outeriño S, Rodríguez M, Pascual M, Graña J. 2005. Efectividad de la intervención educativa en cuidadores de pacientes dependientes en diálisis y valoración de la carga. Rev Soc Esp Enferm Nefro 8(2):156-165.

10. Bermejo F, Rivera J, Pérez F. 1997. Aspectos familiares y sociales en la demencia. Med Clín (Barc) 109:140-146.

11. López J, Crespo M. 2007. Intervenciones con cuidadores de familiares mayores dependientes: una revisión. Psicothema 19(1):72-80.

12. Pinto N, Barrera L, Sánchez B. 2005. Reflexiones sobre el cuidador a partir del programa "Cuidando al cuidador". Revista Aquichan 5(1):128-137.

13. Espín AM. 2009. "Escuela de Cuidadores" como programa psicoeducativo para cuidadores informales de adultos mayores con demencia. Rev Cubana Salud Pública 35(2).

14. Lam Díaz RM, Hernández Ramírez P. 2008. Los términos: eficiencia, eficacia y efectividad ¿son sinónimos en el área de la salud? Rev Cubana Hematol Inmunol Hemoter 24(2).

15. Consejo de Organizaciones Internacionales de las Ciencias Médicas (CIOMS). 1993. Éticas Internacionales para la Investigación y Experimentación Biomédica en Seres Humanos. Ginebra: 53-56.

16. Espín AM, Seco T. 1998. Metodología de intervención educativo-terapéutica en cuidadores de ancianos con demencia de Alzheimer. Rev Arg Geriatr 18:18-27.

17. Donabedian A. 1992. Garantía y monitoría de la calidad de la atención médica. México: Editorial: Perspectivas en Salud Pública.

18. Montorio I, Fernández MI, López A, Sánchez M. 1998. La entrevista de carga del cuidador. Utilidad y validez del concepto de carga. Anales de psicología 14 (2):229-248.

19. Beck A, Steer R, Brown G. 2004. Inventario de depresión de Beck - Segunda edición (BDI-II). Argentina: Editorial Paidós.

20. Vilalta J, Lozano M, Llinás R, López S. 1999. Neuropsychiatric inventory. Propiedades psicométricas de su adaptación al español. Rev Neurol 29(1).

21. Martín M, Merlos MI, Mozos A. 1999. Evaluación de una experiencia grupal de educación para la salud a cuidadores principales de personas con deterioro cognitivo. Trabajo Social Hoy 23:93-105.

22. Toseland RW, Mc Callion P, Smith T, Banks S. 2004. Supporting Caregivers of Frail Older Adults in an HMO Setting. American Journal of Orthopsychiatry 74(3):349-364.

23. Mittelman MS, Roth DL, Coon DW, Haley WE. 2004. Sustained benefit of supportive intervention for depressive symptoms in caregivers of patients with Alzheimer' Disease. American Journal of Psychiatry 161(5):850-856.

24.Schulz R, Gallagher D, Haley W. 2000. Understanding the interventions process: a theoretical/conceptual framework for interventor approaches to caregiving. En Schulz, R. Handbook on Dementia Caregiving. Evidencebased interventions for family caregivers. New York. Springer: 33-60.

25. Crespo M, López J, Zarit S. 2005. Depression and anxiety in primary caregivers: A comparative study of caregivers of demented and nondemented older persons. International Journal of Geriatric Psychiatry 20:591-592.

26. Schumcher KL, Stewart BJ, Archbold PG, Dodd MJ, Dibble SL. 2000. Familia Caregiving Skill: Development of the concept. Res Nurs Health 23(3):191-203.

27. Losada A, Montorio I. 2005. Análisis de las intervenciones con cuidadores familiares de personas con demencia ¿una cuestión de método y no de eficacia? Aten Primaria 35:112.

28. Espín AM. 2008. Evaluación de un programa psicoeducativo para cuidadores informales de adultos mayores con demencia. Perspectivas en Psicología 5(2):93-99.

29. López J, Crespo M. 2007. Intervenciones con cuidadores de familiares mayores dependientes: una revisión. Psicothema. 19(1):72-80.

30. Losada A, Izal M, Montorio I, Márquez M, Pérez G. 2004. Eficacia diferencial de dos intervenciones psicoeducativas para cuidadores de familiares con demencia. Rev Neurol 38(8):701-708.

31. Losada, A. 2005. Influencia de los pensamientos disfuncionales sobre el cuidado en el malestar psicológico de cuidadores de personas mayores con demencia. Resultados de un estudio transversal y de intervención (tesis doctoral). Universidad Autónoma de Madrid.

32. López J. 2005. Entrenamiento en manejo del estrés en cuidadores de familiares mayores dependientes: desarrollo y evaluación de la eficacia de un programa (tesis doctoral) Universidad Complutense de Madrid.

33. Paz F. 2004. Características y necesidades del cuidador primario en las enfermedades neurodegenerativas. Diseño y evaluación de un programa de intervención (tesis doctoral). Universidad Nacional Autónoma de México.

34. Sánchez P; Mouronte P, Olazarán J. 2001. Beneficios de un programa de formación del cuidador en la demencia: experiencia piloto desde la enfermería especializada. Rev Neurol 33:422-424.

35. Gandoy M, Millam JC, García B. 1999. Evaluación y resultados de la instauración de un programa de terapia de grupo dirigido a 
un colectivo de cuidadores familiares de enfermos de Alzheimer. Intervención Psicosocial 8:109-116.

36. Millán JC, Gandoy M, Antelo M. 2000. Helping the family carers of Alzheimer's patients from theory to practice. A preliminary study. Arch Gerontol Geriatrics 30:131-138.

37. Etxeberría I, Yanguas JJ, Buiza C. 2006. Eficacia de un programa psicosocial con cuidadores de enfermos de Alzheimer: análisis de resultados al año de seguimiento. Rev Esp Geriatr Gerontol 40(Supl 3):46-54.
38. Hebert R, Levesque 1, Vezina J. 2003. Efficacy of a psychoeducative group program for caregivers of demented persons living al home: a randomized controlled trial. $J$ Gerontol 58:58-67.

39. Barrera L, Pinto N, Sánchez B. 2006. Evaluación de un programa para fortalecer a los cuidadores familiares de enfermos crónicos. Rev de Salud Pública 8(2):1-8.

40. García N, Pérez MD. 2005. Implantación de un programa de apoyo al cuidador del anciano dependiente hospitalizado. Nure Investigación 7:2-15. 


\title{
POSGRADOS CLÍNICOS
}

Universidad

Surcolombiana

\section{ESPECIALIZACIÓN EN ANESTESIOLOGÍA Y REANIMACIÓN}

Registro ICFES No. 111456170964100111100

Creada mediante acuerdo Consejo Superior Universitario No. 029 del 08-1996

\author{
Denominación Académica: Programa de Especialización en Anestesiología y Reanimación \\ Modalidad: \\ Presencial - Mixta (Diurna y Nocturna) \\ Duración: \\ Cupos: \\ 3 años (6 semestres) \\ Título: \\ 2 Anuales \\ Especialista en Anestesiología y Reanimación
}

\section{ESPECIALIZACIÓN EN GINECOLOGÍA Y OBSTETRICIA}

Registro ICFES No. 111456180000014111400

Creada mediante acuerdo Consejo Superior Universitario No. 036 del 29-05-1996

\author{
Denominación Académica: Programa de Especialización en Ginecología y Obstetricia \\ Modalidad: \\ Presencial - Mixta (Diurna y Nocturna) \\ Duración: \\ Cupos: \\ 3 años (6 semestres) \\ Título: \\ 2 Anuales \\ Especialista en Ginecología y Obstetricia
}

\section{ESPECIALIZACIÓN EN MEDICINA INTERNA}

Registro ICFES No. 111456160004100111400

Creada mediante acuerdo Consejo Superior Universitario No. 037 del 29-05-1996

Denominación Académica: Programa de Especialización en Medicina Interna

Modalidad:

Presencial - Mixta (Diurna y Nocturna)

Duración:

Cupos:

3 años (6 semestres)

Título:

1 Anual

Especialista en Medicina Interna 